\title{
Tutoriales de Youtube como estrategia de aprendizaje no formal en estudiantes universitarios
}

\author{
Youtube tutorials as a non-formal learning strategy for university students
}

\section{Tutoriais do Youtube como estratégia de aprendizagem não formal para estudantes universitarios}

\author{
Ernesto Roque Rodríguez \\ Universidad del Valle de Atemajac, México \\ ernesto.roque.rodriguez@gmail.com \\ https://orcid.org/0000-0003-0067-7137
}

\section{Resumen}

Los videotutoriales de Youtube se han convertido en un recurso necesario para el aprendizaje de los estudiantes. Por ello, el objetivo de este artículo fue conocer y analizar las razones del uso de esos recursos como estrategia de aprendizaje no formal en estudiantes universitarios. Se trata de un estudio mixto secuencial explicativo realizado en dos fases: una cuantitativa y otra cualitativa. Con un muestreo no probabilístico accidental en el que participaron 141 estudiantes, se usó una entrevista grupal para recibir retroalimentación y comentarios. Se realizó un análisis descriptivo a través de las técnicas de contenido y comparación constante. Los resultados demuestran que el $100 \%$ de los estudiantes emplea los videotutoriales de Youtube para una mejor comprensión de un tema (66\%), porque son de fácil acceso y ahorran tiempo (57.4\%), para tener una guía de cómo hacer algo (53.2 \%), para obtener diferentes puntos de vista $(17.7 \%)$ y por experiencia en Youtube $(3.5 \%)$. Se concluye, por tanto, que los videotutoriales pueden ser usados para promover el aprendizaje autónomo, así como el pensamiento reflexivo. 


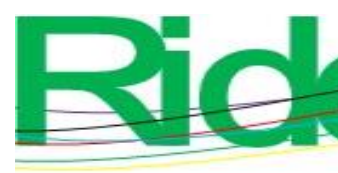

Revista Iberoamericana para la Investigación y el Desarrollo Educativo ISSN 2007-7467

Palabras claves: aprendizaje informal, aprendizaje visual, educación formal, educación, tecnología de la información.

\section{Abstract}

YouTube tutorial videos have become a necessary resource for student learning. The objective of this article was to know and analyze the reasons for the use of YouTube tutorials as a non-formal learning strategy in university students. It is a mixed explanatory sequential study carried out in two phases: quantitative and qualitative. With an accidental nonprobabilistic sampling in which 141 students participated. A group interview was used to receive feedback and comments. A descriptive analysis and analysis is carried out through content and constant comparison techniques. 100\% of the students claimed to use YouTube tutorials: $66 \%$ for a better understanding of a topic, $57.4 \%$ easy access and time saving, $53.2 \%$ have a guide on how to do something, $17.7 \%$ get different points of view and 3.5\% YouTube experience. It is concluded that YouTube tutorials have become non-formal learning tools and promote reflective thinking.

Keywords: education, formal education, informal learning, information technology, visual learning.

\section{Resumo}

Os tutoriais em vídeo do Youtube se tornaram um recurso necessário para o aprendizado dos alunos. Portanto, o objetivo deste artigo foi conhecer e analisar os motivos da utilização desses recursos como estratégia de aprendizagem não formal em estudantes universitários. É um estudo explicativo sequencial misto realizado em duas fases: uma quantitativa e outra qualitativa. Com uma amostragem não probabilística acidental na qual 141 alunos participaram, uma entrevista em grupo foi usada para receber feedback e comentários. Realizou-se análise descritiva por meio de técnicas de conteúdo e comparação constante. Os resultados mostram que $100 \%$ dos alunos utilizam os vídeos tutoriais do YouTube para entender melhor um tema (66\%), por serem de fácil acesso e economizar tempo $(57,4 \%)$, para ter um guia de como fazer $(53,2 \%)$, para obter diferentes pontos de vista $(17,7 \%)$ e da experiência no YouTube $(3,5 \%)$. Conclui-se, portanto, que os vídeos tutoriais podem ser utilizados para promover a aprendizagem autônoma, bem como o pensamento reflexivo. 


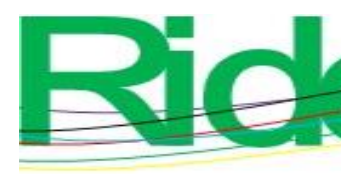

Revista Iberoamericana para la

Investigación y el Desarrollo Educativo

ISSN $2007-7467$

Palavras-chave: aprendizagem informal, aprendizagem visual, educação formal, educação, tecnologia da informação.

Fecha Recepción: Julio 2020

Fecha Aceptación: Diciembre 2020

\section{Introducción}

En el mundo de la globalización y de la sociedad del conocimiento, el acelerado desarrollo de las tecnologías de la información y comunicación (TIC) ha impactado las formas en que interactuamos, accedemos a la información, compramos, protestamos, entre otras. El gran desarrollo de la virtualidad en todas las áreas y el rápido acceso a la información y conocimiento ha provocado en las nuevas generaciones lo que Bauman (2008) llama síndrome de la impaciencia. De acuerdo con este autor, "toda demora, dilación o espera se ha transformado en un estigma de inferioridad" (p. 22).

Las nuevas generaciones de estudiantes millennials han integrado las tecnologías a sus formas de vida cotidiana, y demandan tener respuestas inmediatas a sus inquietudes a través de sus dispositivos digitales. Los millennials — por ser nativos digitales (Prensky, 2001) — son altamente competentes en el manejo de medios tecnológicos. Ellos utilizan las redes sociales de manera frecuente y constante, y pasan horas frente a algunos dispositivos tecnológicos comunicándose con sus amistades, pero desconocen el uso educativo de ese recurso (González, Valdivieso y Velasco, 2020).

Lo anterior ha obligado a las instituciones de educación a incorporar a sus procesos de enseñanza nuevas formas y modelos educativos centrados en el estudiante y en el aprendizaje, en los cuales las herramientas tecnológicas han jugado un rol protagónico. De acuerdo con la Organización de las Naciones Unidas para la Educación, la Ciencia y la Cultura (Unesco) (2017), las TIC apoyan el aprendizaje a lo largo de la vida, ya que han demostrado su capacidad para mejorar el acceso a los programas de educación no formal. Las TIC son uno de los medios por los que se transmiten competencias para la vida práctica y la capacitación laboral en programas de educación no formal. Este tipo de programas generalmente está orientado a ayudar a jóvenes y adultos a encontrar empleo y adquirir autonomía en la sociedad de la información sin la necesidad de asistir de manera formal a una institución educativa. 

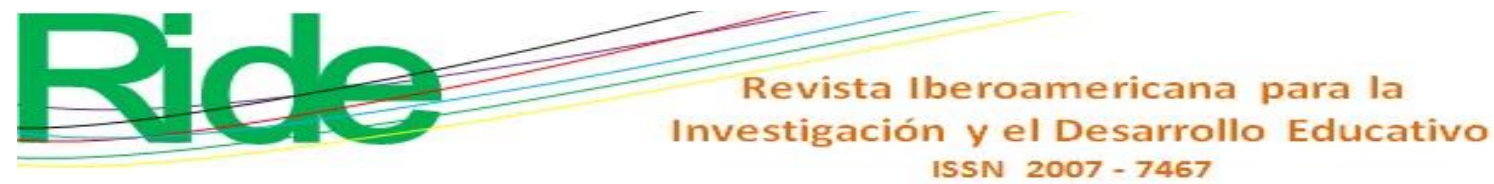

La educación no formal — - según la Unesco (2007) — se refiere al conjunto de actividades educativas organizadas, por regla general, fuera del sistema convencional, aunque con objetivos de aprendizaje claros en función de su duración y la posibilidad de otorgar una certificación de los aprendizajes adquiridos. Para Chacón-Ortiz (2015) la educación no formal es entendida como una actividad socializadora con autonomía curricular, lo que le permite ser dinamizadora y con procesos de formación adaptados a realidades específicas.

La contraparte de la educación no formal es la formal. Esta última —según Celorio y López de Munain (2007) - se estructura de forma jerárquica, con un carácter oficial, reglado, obligatorio, credencialista y ejercida por enseñantes profesionales. La educación informal, en cambio, se refiere al aprendizaje que se efectúa en la vida diaria sin objetivos claramente establecidos. Las personas la adquieren por su experiencia diaria e influencias de la familia, vecinos, compañeros de trabajo, medios informativos, etc.

La proliferación y desarrollo de las tecnologías ha sido tal que en el caso de México el uso de internet, teléfonos inteligentes y redes sociales ha aumentado de manera significativa. De acuerdo con la Encuesta Nacional sobre Disponibilidad y Uso de las Tecnologías de la Información en los Hogares (ENDUTIH, 2018), 74.3 millones de personas de seis años o más utilizan internet. Considerando el número de habitantes de la última encuesta intercensal (2015) en México, igual a 121005 816, significa que representa más de $61.4 \%$ de la población total del país.

Continuando con la ENDUTIH (2018), el número de usuarios de teléfonos inteligentes fue de 83.1 millones en 2018. El uso de Internet se encuentra mayoritariamente extendido entre los jóvenes de 18 a 34 años, con $37.6 \%$ respecto a la población total de seis años y más. En cuanto a las principales actividades de los internautas mexicanos, se pueden mencionar las siguientes: entretenimiento $90.5 \%$, comunicarse $90.3 \%$, obtener información $86.9 \%$, apoyar la educación/capacitación $83.6 \%$, acceder a contenidos audiovisuales 78.1 $\%$, acceder a redes sociales $77.8 \%$, descargar software $49.2 \%$, leer periódicos, revistas o libros $48.7 \%$, interactuar con el gobierno $31.0 \%$, ordenar o comprar productos $19.1 \%$, y efectuar operaciones bancarias en línea $15.4 \%$.

En lo que respecta a medios audiovisuales, la numeraria de Youtube (2019) afirma que más de dos mil millones de usuarios utilizan su plataforma, lo que representa casi la tercera parte de los internautas a nivel mundial. Diariamente los usuarios de Youtube 


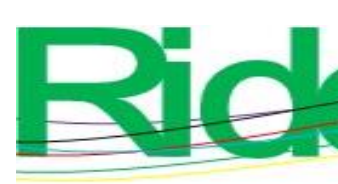

Revista Iberoamericana para la Investigación y el Desarrollo Educativo ISSN 2007-7467

visualizan mil millones de horas de videos, lo que genera miles de millones de vistas. Solo en su versión móvil, llega a más personas de entre 18 y 34 años de edad (lo que no consigue ningún proveedor de televisión por cable de EE. UU.). Además, está localizado en más de 100 países y se encuentra disponible en 80 idiomas.

Youtube es un servicio gratuito de almacenamiento, administración y difusión de videos mediante una cuenta de registro. Los usuarios y visitantes pueden subir, buscar, ver y descargar — gracias a herramientas libres — material en cualquier formato de video o audio. En general, Youtube es un punto de encuentro para quienes quieren exhibir y ver un video, lo que resulta favorable para realizar actividades de enseñanza y de aprendizaje en línea (Ramírez, 2016).

Según Riquelme (27 de septiembre de 2019), México ocupa el tercer lugar en el mundo en consumo de videos de Youtube. El perfil del consumidor es principalmente de millennials, aunque no es un medio específico para jóvenes, y los internautas mexicanos son de los más participativos a nivel global.

En cuanto a los antecedentes de los videotutoriales de Youtube, no hay un dato preciso sobre cuándo aparecieron; lo único que se sabe es que el primer video se subió a la plataforma el 23 de abril de 2005, el cual fue titulado Me at the zoo (Yo en el zoológico). En este aparece Jawed Karim, uno de los creadores de la plataforma. Sin embargo, este video no se pude considerar como un tutorial, ya que su propósito no consiste en enseñar la preparación o la construcción de algo.

En efecto, un videotutorial — de acuerdo con Rodenas (2012) - es una herramienta que muestra paso a paso los procedimientos a seguir para elaborar una actividad, lo cual puede facilitar la comprensión de los contenidos más difíciles. Además, al estar disponible en cualquier momento, se puede recurrir a él cuando se desee y tantas veces como sea necesario. Hoy en día los videotutoriales se han convertido en uno de los mejores recursos educativos, independientemente de la especialidad en la que se empleen.

El objetivo de este artículo, por tanto, es conocer y analizar las razones del uso de los tutoriales de Youtube como estrategia de aprendizaje no formal en estudiantes universitarios. 


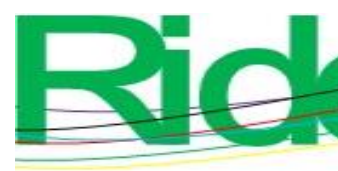

Revista Iberoamericana para la Investigación y el Desarrollo Educativo ISSN $2007-7467$

\section{Metodología}

Esta investigación se centró en una experiencia concreta, vivida por los estudiantes de la licenciatura en Administración Gubernamental y Políticas Públicas (LAGPP) de la Universidad de Guadalajara (UDG). La pregunta de investigación fue la siguiente: ¿cuáles son las razones del uso de los tutoriales de Youtube como estrategia de aprendizaje no formal en los estudiantes universitarios?

Para intentar hallar respuestas, se efectuó un estudio mixto secuencial explicativo realizado en dos fases (Creswell y Creswell, 2018). La primera fue cuantitativa y consistió en una encuesta en línea enviada a todos los estudiantes por Google Drive. La segunda fue cualitativa y consistió en una entrevista focal realizada a un número más reducido de participantes, a los que se les dieron a conocer los resultados de la primera fase, y se les solicitaron sus comentarios.

El tipo de muestra que se utilizó para la fase cuantitativa fue un muestreo no probabilístico accidental, el cual —según Otzen y Manterola (2017) — recluta casos de manera casual por algún medio hasta completar el número de sujetos deseados. Se logró encuestar a 141 estudiantes de la LAGPP, esto es, $27.86 \%$ de una población de 506 estudiantes matriculados en el calendario 2017A, de acuerdo con la estadística institucional de la UDG (2017). En la fase cualitativa el muestreo que se utilizó fue intencional basado en criterios, en el cual participaron 11 estudiantes. Los criterios fueron dos: ser estudiante activo de la LAGPP, y haber contestado la encuesta en línea.

El cuestionario tipo encuesta se diseñó con base en una revisión de la literatura sobre los usos de Youtube para fines educativos; además, se consultó a tres expertos en el área de TIC aplicadas al aprendizaje y se piloteó con 10 estudiantes que contestaron el instrumento antes de enviarlo a través del Google Drive.

La encuesta en línea se aplicó durante el mes de octubre y la primera semana de noviembre de 2017. Los datos se trabajaron en el SPSS y las gráficas se diseñaron en Excel. Posteriormente, se realizó la entrevista grupal (focus group) durante la última semana de noviembre en la que participaron 11 estudiantes, los cuales manifestaron que querían conocer los resultados de la fase cuantitativa.

El análisis de la información para la encuesta en línea fue estadístico de carácter descriptivo, en el que se utilizó SPSS y Excel para un mejor diseño de gráficos. El análisis de la entrevista focal fue realizado a través de las técnicas de análisis de contenido y 

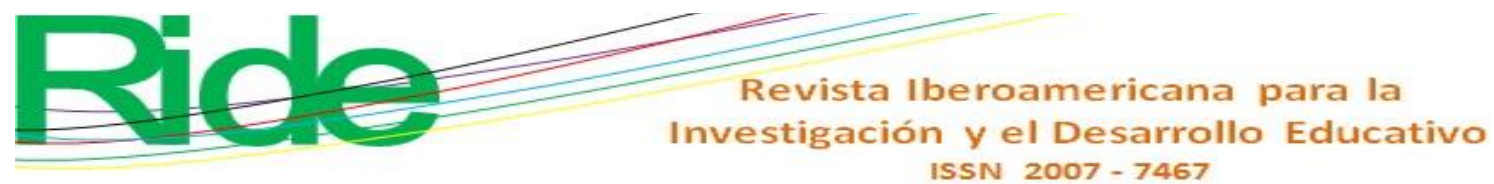

comparación constante (Meriam y Tisdell, 2016), que se utilizó para reafirmar e iluminar los resultados cuantitativos por parte de los participantes.

\section{Resultados}

Habiendo analizado toda la información recabada, se puede afirmar que los estudiantes hacen un uso significativo de los tutoriales que se encuentran en Youtube para complementar sus conocimientos como una estrategia de aprendizaje autodidacta. A continuación, se muestran los resultados de la encuesta aplicada en línea, seguidos de los testimonios más relevantes de la entrevista focal realizada a los participantes.

El perfil sociodemográfico de los alumnos quedó distribuido de la siguiente manera: $59.6 \%$ fueron mujeres y $40.5 \%$ hombres; la edad promedio fue de 22 años. Todos ellos estudiantes de licenciatura en Administración Gubernamental y Políticas Públicas.

Las motivaciones de uso de Youtube quedaron representadas de la siguiente manera: $91.5 \%$ de los estudiantes utilizan esta plataforma para entretenimiento y aprendizaje; $5.7 \%$ solamente para entretenimiento, y $2.8 \%$ para aprendizaje.

Figura 1. Motivaciones de uso de Youtube

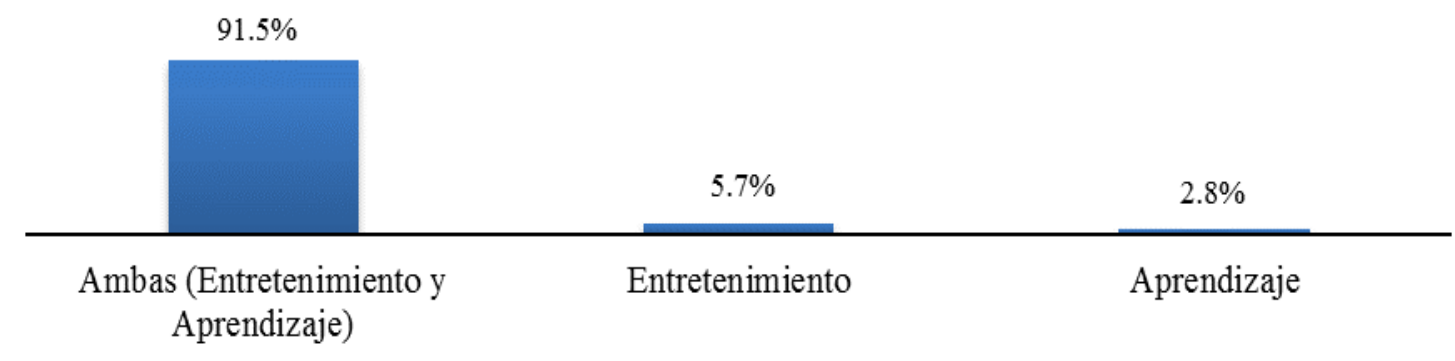

Fuente: Elaboración propia

En el grupo focal, los 11 participantes concordaron con los resultados expuestos. Cuando se les preguntó sobre si no se estaría abusando del uso del Youtube y sus tutoriales en sustitución de los libros y fuentes impresas, un participante comentó: "No, porque solo los uso para reforzar la información y complementar los temas vistos en clase" (participante 6). Esta percepción fue respaldada por el resto de los asistentes al grupo focal.

Lo anterior concuerda con Bacescu (2017), quien realizó un estudio sobre Blogging de entretenimiento y educativo, en el cual reporta que $83 \%$ de sus participantes afirmaron que usaban esa plataforma como herramienta de aprendizaje. Asimismo, en otro estudio 


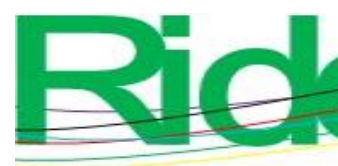

Revista Iberoamericana para la
Investigación y el Desarrollo Educativo
ISSN $2007-7467$

sobre percepciones de Youtube realizado por Flalinger y Owen (2009), se determinó que $73 \%$ de los participantes usa esta plataforma como apoyo para el aprendizaje.

Gallego y Murrillo (2018) realizaron un estudio para conocer las predisposiciones de los estudiantes de grado en la Facultad de Educación de la Universidad de Sevilla, España, para utilizar Youtube como herramienta complementaria en la práctica docente. Los autores encontraron que para $92.7 \%$ dicho portal resultaba útil debido a que era un elemento innovador (40\%), servía como fuente de información (20\%), ampliaba los contenidos (19.1 $\%)$, afianzaba conocimientos (12.2\%) e integraba las TIC en el aula (8.7\%).

Volviendo a nuestra investigación, y sobre la frecuencia de uso de esta plataforma, $36.9 \%$ la utiliza de dos a tres veces por semana, 32.6\% una vez a la semana, $19.9 \%$ una vez al mes y $10.6 \%$ diariamente.

Figura 2. Frecuencia de uso de Youtube

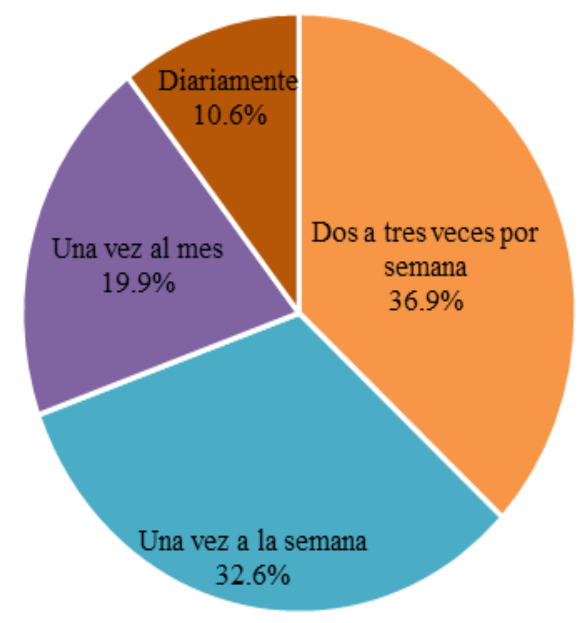

Fuente: Elaboración propia

En la entrevista focal, $73 \%$ de los participantes afirmaron que usaban Youtube diariamente y $27 \%$ de dos a tres veces por semana. Esto demuestra una tendencia mayor en los participantes de la entrevista focal que en quienes contestaron la encuesta en línea. A los primeros se les preguntó si se estaban cambiando los libros por los videotutoriales. El participante 6 respondió que no, porque solo se usaban para reforzar la información o para complementar un tema que no se había entendido en clase. 


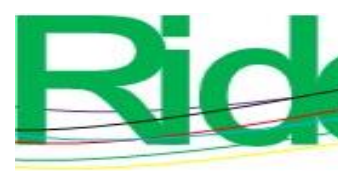

Revista Iberoamericana para la Investigación y el Desarrollo Educativo ISSN 2007 - 7467

En un estudio de Statista (2015) realizado en España en 2015, relacionado con la frecuencia de uso de Youtube, $40 \%$ de los participantes comentó que utilizaba ese recurso una vez a la semana, mientras que 20 \% señaló que una vez al día. Estos resultados son muy parecidos a los obtenidos en nuestra encuesta.

La Asociación de Internet MX (AMPICI) — en su estudio sobre los hábitos de los usuarios de Internet en México 2018 - menciona que ese recurso se emplea principalmente para acceder a redes sociales (89 \%). De hecho, las más empleadas son Facebook (98\%), Whatsapp (92\%) y Youtube (82\%).

En relación con la frecuencia de uso de los videotutoriales de Youtube, todos los estudiantes encuestados dijeron que los empleaban en algún momento. En concreto, $40.4 \%$ los utilizaba de dos a tres veces por semana, 33.3\% una vez a la semana, $19.1 \%$ una vez al mes y $6.4 \%$ diariamente.

Figura 3. Frecuencia de uso de videotutoriales de YouTube

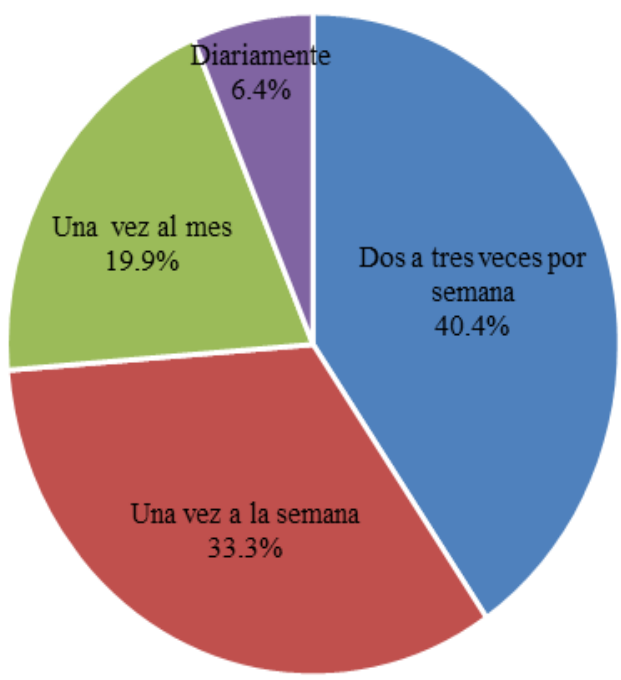

Fuente: Elaboración propia

En la entrevista focal, $9 \%$ de los participantes afirmaron usarlos diariamente, $18 \%$ una vez por semana y $73 \%$ de dos a tres veces por semana. Esto demuestra también una tendencia mayor en los participantes de la entrevista focal. Por otra parte, y en cuanto a cuáles podrían ser los peligros del abuso de los videotutoriales, el participante 11 manifestó: "No es un abuso si se cuida el tiempo de uso. En todo caso, el abuso siempre ha existido, pero en diferente medio. Décadas atrás se decía lo mismo de la televisión”. 


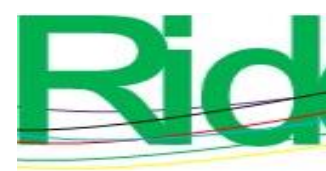

Revista Iberoamericana para la
Investigación y el Desarrollo Educativo
ISSN $2007-7467$

Las razones por las que los estudiantes veían los videotutoriales de Youtube fueron las siguientes: para una mejor comprensión de un tema (66 \%), fácil acceso y ahorro de tiempo (57.4\%), tener una guía de cómo hacer algo (53.2\%), obtener diferentes puntos de vista $(17.7 \%)$, experiencia del youtuber $(3.5 \%)$.

Figura 4. Razón de uso de los videotutoriales de Youtube

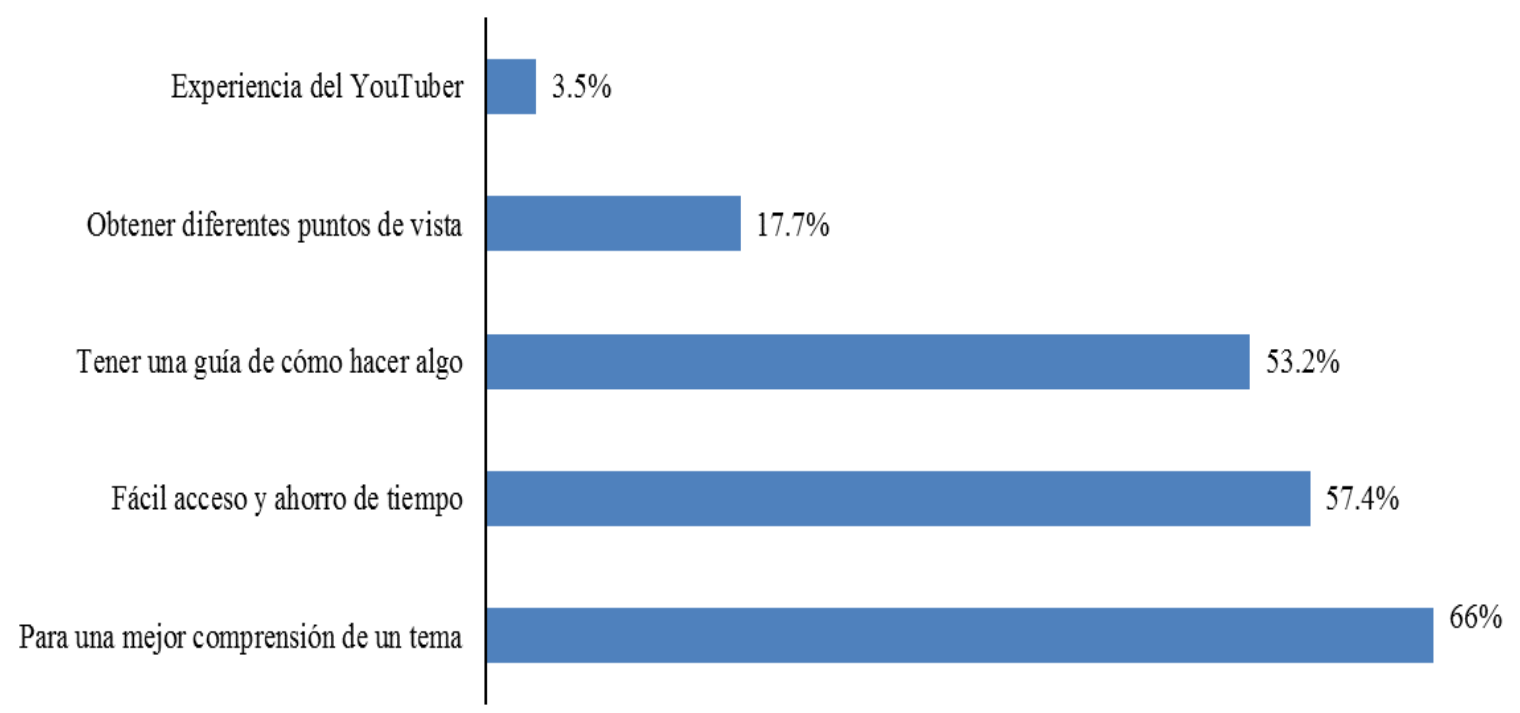

Fuente: Elaboración propia

Las razones de uso de los participantes en la entrevista focal fueron aclarar dudas de la escuela, específicamente relacionadas con matemáticas. En este punto, la participante 8 dijo que recurría a los videotutoriales porque en ellos comprendía más que cuando le explicaba su profesor de aula. Asimismo, estos participantes expresaron que los empleaban para aprender inglés, manejo de redes sociales, ventas (marketing), aplicaciones para el celular y automóviles. El participante 3 agregó que los videotutoriales se pueden descargar y escuchar mientras viajaba. Por su parte, el participante 6 comentó que los tutoriales se pueden repetir infinidad de veces. En general, los participantes estuvieron de acuerdo con los resultados de la encuesta. Al preguntarles sobre el medio utilizado para ver los videotutoriales, $71.6 \%$ se refirió al smartphone, $24.8 \%$ computadora personal y $3.5 \%$ tableta. 


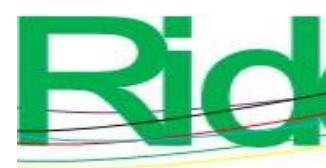

Revista Iberoamericana para la Investigación y el Desarrollo Educativo ISSN 2007-7467

Figura 5. Medios usados para ver los videotutoriales de Youtube

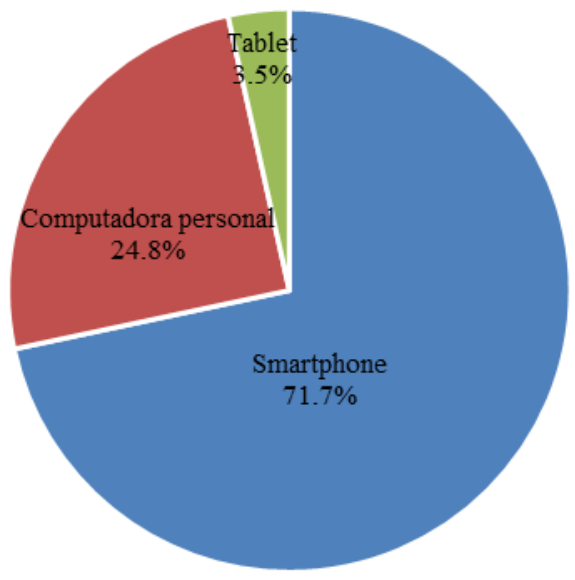

Fuente: Elaboración propia

Ocho de los 11 participantes del grupo focal contestaron que usaban el smartphone para ver los tutoriales, mientras que tres preferían la computadora. En cuanto a los criterios de selección de un videotutorial de YouTube, 72.3 \% afirmó que la descripción del video es el factor más importante, $55.3 \%$ los selecciona por los comentarios sobre el video, $50.4 \%$ por la duración del video, 44 \% por el número de visitas, $21.3 \%$ por número de likes o dislikes y $21.3 \%$ por la simpatía con el youtuber.

Figura 6. Criterios de selección de un videotutorial de Youtube

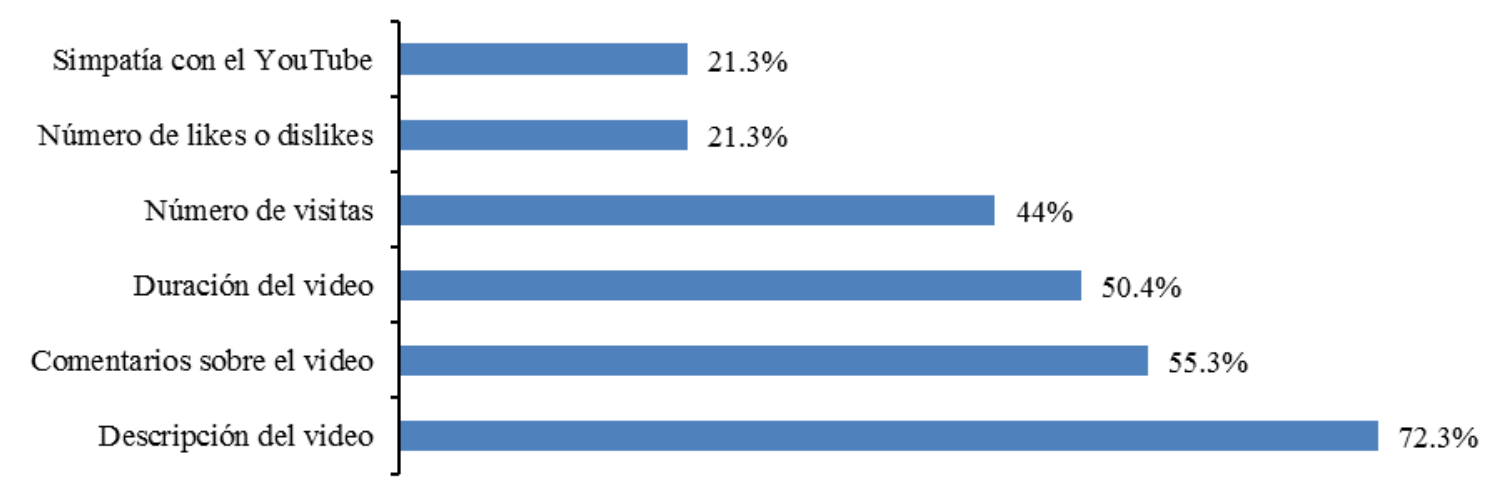

Fuente: Elaboración propia

Al preguntar a los participantes del grupo focal sobre los criterios de la selección del tutorial, $54.5 \%$ afirmaron que lo hacían por la descripción del video, 45 \% por el número de visitas (lo cual indica la calidad del video) y 36 \% la duración del video (quieren videos ágiles y no tediosos). Relacionado con el punto anterior, el participante 3 agregó: "Hay temas que 
no se pueden explicar en dos minutos". El número de likes o dislikes obtuvo $27 \%$ y los comentarios de los videos obtuvieron $18 \%$. Otros criterios menos importantes fueron el título del video, la forma en que se envía el mensaje y la miniatura del video.

Garrett (2016) realizó un estudio descriptivo en Estados Unidos, exclusivamente para el caso de videotutoriales relacionados con el uso de Microsoft Excel, usando como categoría las consultas. Para ello, se apoyó en una base de datos de American On Line (AOL), que contiene 20 millones de consultas de 650000 usuarios. Encontró que los videotutoriales ayudan a resolver problemas relacionados con el uso y manejo, e identificó los criterios de consulta según el siguiente orden: vistas, likes, dislikes, comentarios y duración. Aunado a esto, el autor señaló que la edad tiene una correlación positiva con ver videos de actividades sociales, aunque la duración no se correlacionó con ninguna de las actividades sociales. Los likes, vistas, comentarios y dislikes tuvieron una correlación fuerte con consultas devueltas (regreso al video o ver de nuevo el video), pero no tuvieron conexión con los rankings de búsqueda. El autor concluye que las consultas de tutoriales indican que los aprendices informales en algunas áreas no lo usan óptimamente como una ayuda. Incluso asegura que algunos no saben específicamente cuáles soluciones necesitan.

De acuerdo con Bengochea y Medina (2013), los videotutoriales de corta duración (10 a 15 minutos) o píldoras formativas son pequeñas piezas de material didáctico, creadas como objetos de aprendizaje de contenido audiovisual y diseñadas para complementar las estrategias de formación y facilitar la comprensión de alguna asignatura. Actualmente, muchas universidades graban sus clases y las suben a un canal.

Los videotutoriales se diferencian de los usados en otras plataformas por su duración. Además, para su grabación no se necesitan instrumentos sofisticados. Rabee et al. (2015), en un estudio realizado en una escuela de medicina de Reino Unido, encontraron que los videos de Youtube son recursos que permiten la interacción entre los usuarios mediante los comentarios. Estos autores, sin embargo, también indican que esos videos presentan algunos problemas, como la falta de regulación de su contenido, de ahí que algunos alumnos se confundan sobre cuáles videos pueden ser útiles y cuáles no.

En nuestro estudio encontramos que los tutoriales tienen diferente utilidad; por ejemplo, $91.5 \%$ de los estudiantes los usa como apoyo a tareas y trabajos escolares, $53.9 \%$ para ver formas de cocinar, repostería y coctelería, $47.5 \%$ para ampliar su conocimiento en salud física y mental, $40.4 \%$ para el desarrollo de hardware y software, $39.7 \%$ para aprender 

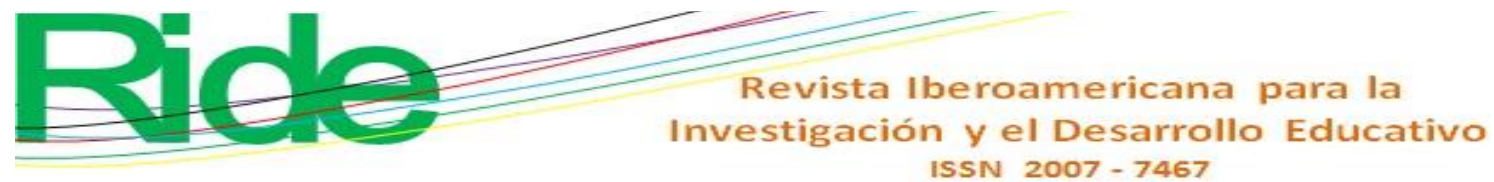

maquillaje y peinado, $38.3 \%$ para actividades vinculadas con el arte, la artesanía y las manualidades, $29.1 \%$ para aprender idiomas, $19.1 \%$ para usos, reparación y armado de electrodomésticos y electrónicos, $17.7 \%$ para crear música o tocar un instrumento, $10.6 \%$ para diseño y reparación de muebles, y $3.5 \%$ para otras actividades (como matemáticas, física y política, entretenimiento, corte y confección de ropa, canto, arreglo personal).

Figura 7. Razones de uso de videotutoriales de Youtube

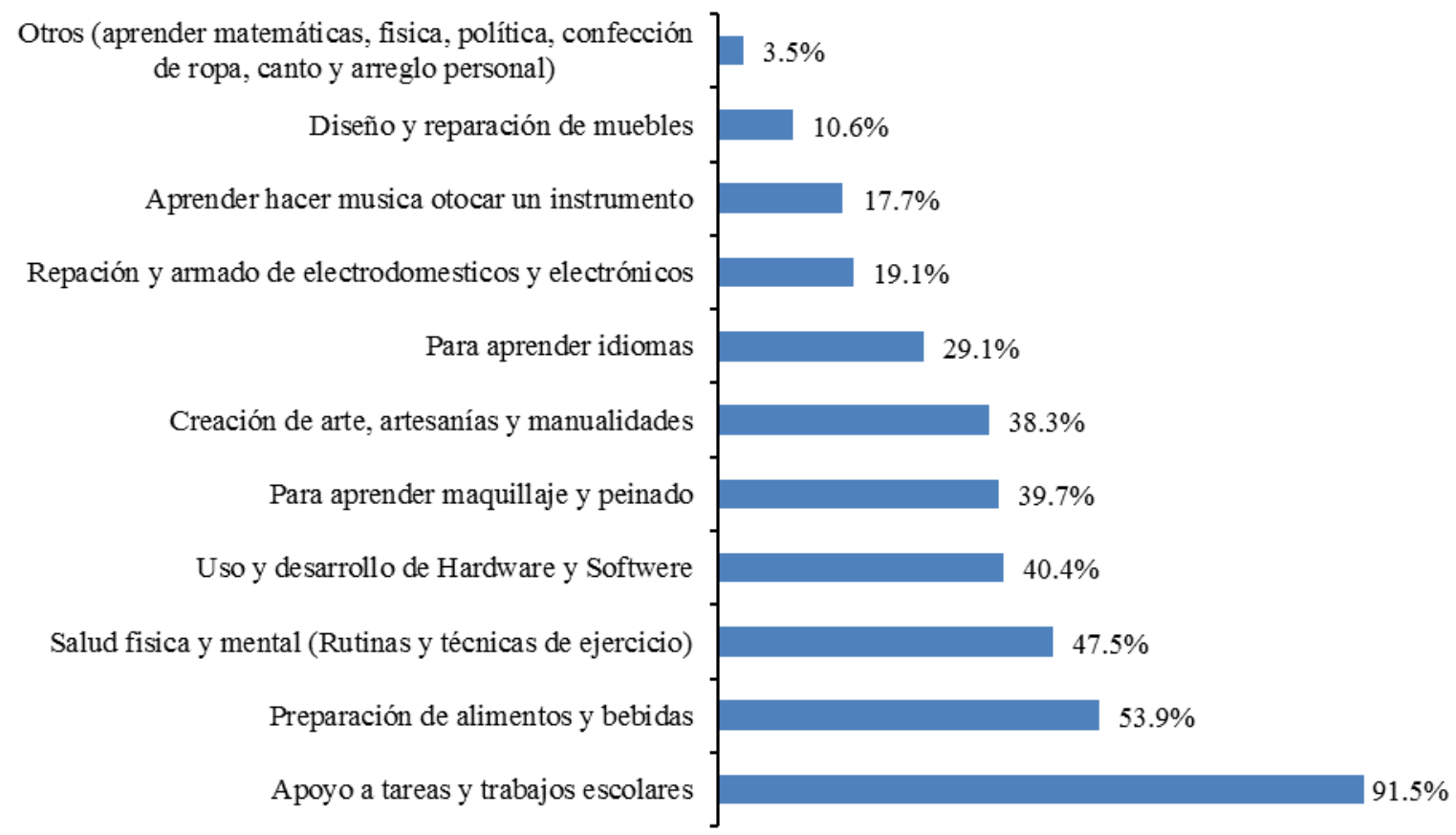

Fuente: Elaboración propia

Siguiendo con las razones de uso de los videotutoriales de Youtube, los participantes del grupo focal indicaron lo siguiente: apoyo a tareas y trabajos escolares (lo que concuerda con los resultados de la encuesta), aprender a preparar alimentos y bebidas, uso de hardware y software, aprender a tocar un instrumento musical, cuidar la salud física y mental, aprender técnicas de venta e idiomas, tener entrenador personal y aprender de jardinería. En términos generales, los participantes concordaron con los resultados cuantitativos.

Según un estudio realizado en España por Julià (5 de junio de 2018) entre personas de 16 a 65 años de edad, Youtube es la tercera red social más usada con 69 \% (superada solo por Facebook y Whatsapp). Esas personas consultadas usan Youtube 10 horas por día y $28 \%$ siguen a influencers. Asimismo, Youtube es la red social usada en magnitudes iguales tanto por la generación Z (año 2001-2020) como por la generación millennials (1980-2000), con 


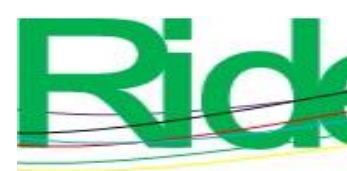

Revista Iberoamericana para la Investigación y el Desarrollo Educativo ISSN $2007-7467$

$75 \%$ y $74 \%$, respectivamente (solo después de Whatsapp y Facebook). Para la generación Z, Youtube es más preferida que para los millennials.

En nuestro estudio, el canal favorito de los estudiantes es Julio profe (con $10.6 \%$ de las preferencias), creado por un docente colombiano que explica contenidos relacionados con física y matemáticas. Además, mencionan otros canales como Luisito comunica, Yuya, El pulso de la república, Chiis Fix, Alicia Soltero medíos, All Recipes Mexixo, Behindthechaiy, Beyonce, Blogilates, Chumel Torres, Clau Reads Books, Craftingeek, Daniel el Tigre, Dionyorki, Dross, El robot de Platón, Enamorándonos, Escorpión dorado, Fernand flo, Galatzi, GuitarraViva (GV), Harmurdog, Izhan, Jonathan Venegas, Juca, Jürgen Klaric, La vanguardia, Loquillo Florez, Marco Antonio Regil, Marisol Pink y Deporlovers, Megaken, Munchies, MYM Alk4pon3, Paula Galindo, Paulette, Sebastian Villalobos, Sol yoga, Talk to me in korean, marketing TV, Quondos, EOI, Tik Tak Draw my life, Topes de gama, Vevo, Vicky receta fácil, Victor tutor y Zares del universo. Es fundamental decir que $46.1 \%$ no tiene un canal favorito.

En la pregunta abierta a todos los participantes de la encuesta se les pidió las razones por las que se visualiza un canal de Youtube o por qué sigue a determinado youtuber. Las respuestas fueron las siguientes: $5.6 \%$ porque presentan temas de fácil comprensión, $3.5 \%$ por diversión, $2.8 \%$ por la calidad de contenido, $2.1 \%$ por aprendizaje. Otras mencionadas fueron porque es un canal práctico y dinámico para aprender un idioma, porque sube entrevistas con expertos o profesionales, para aprender matemáticas, para aprender a hacer cosas en vez de siempre comprarlas, para conocer de nuevas culturas, por conocimientos didácticos y entretenidos, por crecimiento personal y profesional, por la crítica política que presenta, porque da a conocer noticias recientes de manera graciosa, por distracción y diversión, porque muestra nuestras tradiciones, para pasar el rato, para enterarse de noticias contadas de manera divertida y con comedia, porque es muy bueno explicando y aclara todas mis dudas, por la excelente manera de explicar temas relacionados a métodos cuantitativos, para conocer información del acontecer mexicano, para aprender canciones nuevas en guitarra, para entender temas que no quedaron claros en clase, para escuchar música, etc.

Bhatia (2017) realizó un estudio en Estados Unidos sobre 250 videos de 10 youtubers diferentes, para lo cual tomó en cuenta variables como el género, la región y los antecedentes personales. El análisis se enfocó en las identidades sociales y la relación entre los participantes, así como en las creencias discursivas que pueden crear significados. Sus 


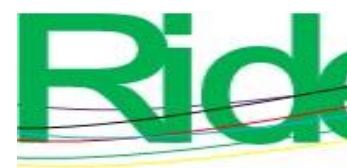

Revista Iberoamericana para la Investigación y el Desarrollo Educativo ISSN 2007 - 7467

resultados demuestran que el aprendizaje a través de los videotutoriales es un estilo de aprendizaje muy importante para la generación NET. Además, los tutoriales de Youtube, generan emociones diferentes a otro tipo de herramientas pedagógicas. El autor agrega que el contenido de Youtube es construido sobre la base de las culturas e identidades (creador, mentor, maestro, estudiante y suscriptor). Para este autor lo importante es construir una identidad en línea de manera profesional, donde los videos sean acompañados por comentarios y subscritores que reflejan la aceptación del video. Bhatia menciona que los tutoriales ayudan en la motivación y alcance de objetivos pedagógicos. Sin embargo, se necesita la creación de una identidad. Además, indica que en los videotutoriales resurge una forma tradicional de establecer "un monólogo pedagógico", en el cual los youtubers lo dibujan como una forma tradicional asociada con la expertiz. El logro o éxito del video depende de la profesionalidad del youtuber para comunicar y enseñar, y eso dependerá de los suscriptores y seguidores que acumule.

Siguiendo con Bathia, para los youtubers la interacción es esencial, pues los participantes no solo se subscriben, sino que interactúan con él e incluso generan contenidos. Los youtubers postean dos a tres videos por semana, sin contar todos los comentarios que realizan en otras redes sociales. Los usuarios analizan los comentarios sobre los videos, aunque no son representativos enteramente de las razones por las que se ven los videos.

\section{Discusión}

Bacescu (2017) menciona en un estudio que Youtube tiene un lado educativo, ya que $83 \%$ lo ha usado como herramienta de aprendizaje. En nuestro caso, encontramos que 91.5 $\%$ de los estudiantes usa dicha plataforma como apoyo para tareas y trabajos escolares. Además, también la utilizan para resolver actividades cotidianas según sus necesidades. Esto tiene implicaciones en términos de incluir en las planeaciones didácticas de las instituciones educativas una gran cantidad de videotutoriales para complementar los materiales y las lecturas de los cursos. Sin embargo, para tomar en cuenta esta sugerencia, se deben valorar aspectos esenciales como la duración y la claridad en las explicaciones. Es recomendable, además, que el docente realice sus propios videotutoriales para que los estudiantes se sientan más seguros de los contenidos que están consultando. El docente como especialista de la asignatura deberá de ser muy selectivo en los videos que proporcione o sugiera para evitar la desviación y desmotivación de los estudiantes; especialmente porque en Youtube existe una 


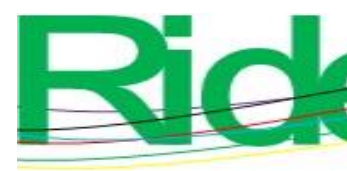

Revista Iberoamericana para la Investigación y el Desarrollo Educativo ISSN 2007-7467

gran cantidad de videotutoriales que pudieran ser publicados por personas que no son especialistas en los temas (Sato, 2012).

Por otra parte, y a pesar de las críticas sobre el uso de los videotutoriales de Youtube, estamos de acuerdo con Flalinger y Owen (2009), quienes sostienen que como herramientas de aprendizaje expanden las habilidades tecnológicas de los estudiantes.

Al respecto, Ruiz y Escurra (2013) encontraron en su trabajo que $68.7 \%$ de sus participantes usa Youtube como entretenimiento, $48.6 \%$ para realizar tareas académicas y $22.2 \%$ para estar al tanto de lo que sucede en el mundo. En nuestro estudio hallamos que los alumnos los emplean para aprender y como entretenimiento. Además, los dispositivos preferidos para consultarlos son el celular $(71.6 \%)$, dato superior al reportado por Bacescu (2017) de $50 \%$.

Gallardo (2013), por su parte, en una indagación realizada en España, encontró que la mayoría de usuarios de esa plataforma son hombres de entre 18 y 34 años, interesados por los contenidos cercanos y locales a su identidad cultural. Asimismo, Davidson, Liebald, Liu, Nandy, y Van Vleet (2010) señalan que los jóvenes son los encargados de recomendar los videos de Youtube.

Tafadzwa Maziriri, Gapa y Chuchu (2020) comentan que para los estudiantes el uso de los videotutoriales de YouTube es muy útil; además, tienen actitudes positivas y favorables por la facilidad de uso y accesibilidad en todo momento, lo cual se diferencia de las barreras comunicativas que algunas veces se establecen entre docentes y profesores.

En cuanto a los criterios didácticos de esta herramienta tecnológica, Lange (2018) considera que no se evalúa su pertinencia ni su calidad pedagógica. Esta es una observación que si bien no fue consultada en nuestra investigación, se debe tomar en cuenta cuando se quiera recomendar algún video en particular. De hecho, se deberían preferir canales conocidos y consolidados. Aun así, este aspecto referente a la calidad didáctica de un video sirve de sustento para formular una interrogante: ¿los likes, dislikes, comentarios o la duración del video son requisitos suficientes para determinar su calidad? Para Lange (2018) los comentarios son esenciales porque representan un mecanismo de retroalimentación para usuarios y creadores en torno a aspectos técnicos y de ejecución.

Por otra parte, se debe enfatizar que los videotutoriales son un complemento, y no el reemplazo de los libros. Además, ambos se pueden consultar las veces que sean necesarias. Si bien Yim, Sturzinger y Lowrance (16 de junio de 2019) encontraron en su trabajo que aún 


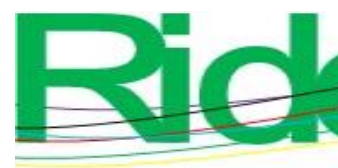

Revista Iberoamericana para la Investigación y el Desarrollo Educativo ISSN 2007-7467

prevalece el uso del libro de texto para consultar algún tema, la realidad demuestra que los alumnos cada vez consumen más información audiovisual para realizar sus labores académicas. De hecho, nuestros hallazgos muestran que los estudiantes usan los videotutoriales porque son más fáciles de digerir.

Por último, vale comentar que una limitante de nuestra investigación fue que se realizó únicamente para un programa universitario. Aun así, consideramos que los resultados pueden ser útiles y referentes para otras indagaciones, por lo que queda pendiente replicarlos en otros programas, modalidades y universidades.

\section{Conclusiones}

Sin lugar a dudas, los videotutoriales de Youtube se han convertido en herramientas de aprendizaje autónomo que promueven el pensamiento reflexivo. De hecho, los estudiantes ven el uso de estos recursos como un complemento o apoyo para sus actividades escolares. Si bien se recomienda que sean los profesores de cada materia quienes creen sus propios videos, también puede suceder que recomienden algunos de los tantos que se hallan publicados en esa plataforma. Para ello, sin embargo, se deben tomar en cuenta criterios relacionados con la calidad didáctica y la duración (de tres a ocho minutos). Esta estrategia no tendrá resistencia por parte de los estudiantes, ya que ellos mismos son quienes la sugieren.

En definitiva, se ha demostrado que los videotutoriales de Youtube como estrategia de aprendizaje no formal son una herramienta pedagógica fundamental que debe ser aprovechada, ya que se puede implementar en todo momento, lugar y cuantas veces se requiera.

Finalmente, no se debe pensar que este apoyo tecnológico sustituye al docente o a los libros recomendados. En su lugar, debe ser concebido como otra herramienta que viene a enriquecer el abanico de estímulos para fomentar la creatividad y el sentido crítico del estudiante. 


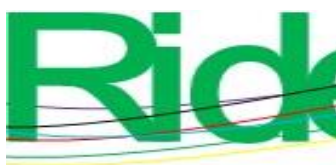

Revista Iberoamericana para la Investigación y el Desarrollo Educativo ISSN 2007 - 7467

\section{Referencias}

Asociación de Internet MX (2018). Estudio sobre los hábitos de los usuarios de internet en México 2018. Recuperado de https://www.asociaciondeinternet.mx/es/component/remository/Habitos-deInternet/14-Estudio-sobre-los-Habitos-de-los-usuarios-de-Internet-en-Mexico2018/lang,es-es/?Itemid=

Bacescu, M. (2017). Blogging, Vlogging- from entertaimente to education. In $13^{\text {th }}$ international scientific conference eLearning \& Software for Education, Bucharest, 27 y 28 de mayo de 2017, 1, 369-376. Doi: 10.12753/2066-026X-17-054

Bauman, Z. (2008). Los retos de la educación en la modernidad líquida. Gedisa.

Bengochea, L. y Medina, J. (2013). El papel de los video tutoriales accesibles en el aprendizaje del futuro. En acta del V Congreso Internacional ATICA, Huencayo, Perú. Recuperado de http://www.esvial.org/wpcontent/files/Videotutoriales_BengocheaMedina.pdf

Bhatia, A. (2017). Interdiscursive performance in digital professions: The case of YouTube tutorials. Journal of Pragmatics, 124, 106-120. Doi: 10.1016/j.pragma.2017.11.001

Celorio, G. y López de Munain, A. (coords.) (2007). Diccionario de educación para el desarrollo. Hegoa. Recuperado de http://libros.metabiblioteca.org/bitstream/001/491/1/diccionario_2.pdf

Chacón-Ortiz, M. (2015). El proceso de evaluación en educación no formal: un camino para su construcción. Revista Electrónica Educare, 19(2), 21-35. Doi: 10.15359/ree.192.2

Creswell, J. W. and Creswell, J. D. (2018). Research design: Qualitative, quantitative and mixed methods approaches. SAGE publications.

Davidson, J., Liebald, B., Liu, J., Nandy, P. and Van Vleet, T. (2010). The YouTube Video Recommendation System. RecSys '10: Proceedings of the fourth ACM conference on Recommender systems, Association for Computing Machinery, New York, NY, USA (pp. 293-296). Doi: 10.1145/1864708.1864770

Flalinger, B. and Owen, R. (2009). YouTube as a Learning Tool. Journal of College Teaching \& Learning, 6(8), 15-28. Doi: 10.19030/tlc.v6i8.1110 


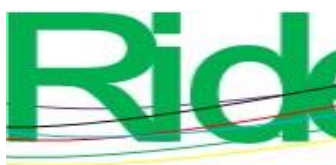

Revista Iberoamericana para la Investigación y el Desarrollo Educativo ISSN 2007 - 7467

Gallardo, J. (2013). Descripción cuantitativa y cualitativa del espectador de videos en YouTube España. Communication Papers, 2(02), 11-22. Doi: 10.33115/udg_bib/cp.v2i02.22102

Gallego, C. y Murillo, P. (2018). La práctica docente mediada con tecnologías. YouTube como herramienta de aprendizaje en la educación superior. Foro Educacional, (31), 11-29. Doi: 10.29344/07180772.31.1827

Garrett, N. (2016). Mapping Self-Guided Learners Searches for Video Tutorials on YouTube. Journal of Educational Technology System, 44(3), 319-331. Doi: $10.1177 / 0047239515615851$

González, C., Valdivieso, L. y Velasco, V. (2020). Estudiantes universitarios descubren redes sociales y edublog como medio de aprendizaje. Revista Iberoamericana de Educación a Distancia, 23(1), 1-17. Doi: 10.5944/ried.23.1.24213

Instituto Nacional de Estadística y Geografía (Inegi) (2018). Encuesta Nacional sobre Disponibilidad y Uso de Tecnologías de la Información en los Hogares (ENDUTIH). Recuperado de https://www.inegi.org.mx/programas/dutih/2018/

Instituto Nacional de Estadística y Geografía (Inegi) (2019). Encuesta intercensal 2015. Recuperado de https://www.inegi.org.mx/programas/intercensal/2015/

Julià, V. (5 de junio de 2018). Estudio Anual de Redes Sociales 2018. Recuperado de https://blog.elogia.net/estudio-anual-de-redes-sociales-2018

Lange, P. (2018). Informal Learning on YouTube. The International Encyclopedia of Media Literacy, 1-11. Doi: 10.1002/9781118978238.iem10090

Meriam, S. and Tisdell, E. (2016). Qualitative Research, a gruide to desing and implementation, Jossey-Bass.

Organización de las Naciones Unidas para la Educación, la Ciencia y la Cultura (Unesco). (2007). Informe de seguimiento de la educación para todos en el mundo: bases sólidas. Recuperado de http://www.unesco.org/education/GMR/2007/es/ESP_complet_2007.pdf

Organización de las Naciones Unidas para la Educación, la Ciencia y la Cultura (Unesco). (2017). Educación no formal. Recuperado de http://www.unesco.org/new/es/unesco/themes/icts/lifelong-learning/non-formaleducation/ 


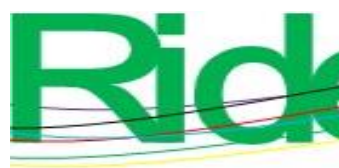

Revista Iberoamericana para la Investigación y el Desarrollo Educativo ISSN $2007-7467$

Otzen, T. y Manterola, C. (2017). Técnicas de muestreo sobre una población a estudio. International Journal of Morphology, 35(1), 227-232. Doi: 10.4067/S071795022017000100037

Prensky, M. (2001). Digital Natives, Digital Immigrants. On the Horizont, 9(5), 1-6. Retrieved from https://www.marcprensky.com/writing/Prensky\%20\%20Digita1\%20Natives,\%20Digita1\%20Immigrants\%20-\%20Part1.pdf

Rabee, R., Najim, M., Sherwani, Y., Ahmed, M., Ashraf, M., Al-Jibury, O., Rabee, N., Najim, R. and Ahmed, A. (2015). YouTube in medical education: A student's perspective. Medical Education Online, 20(1), 1- 2. Doi: 10.3402/meo.v20.29507

Ramírez, M. (2016). Posibilidades del uso educativo del YouTube. Ra Ximhai, 12(6), 537546. Recuperado de https://www.redalyc.org/pdf/461/46148194036.pdf

Riquelme, R. (27 de septiembre de 2019). Youtube tiene 19\% del mercado de consumo de video en México. El Economista. Recuperado de http://eleconomista.com.mx/tecnociencia/2015/08/20/mexico-tercer-lugar-consumovideo-youtube

Rodenas, M. (2012). La utilización de los videos tutoriales en educación. Ventajas e inconvenientes. Software gratuito en el mercado. Revista Digital Sociedad de la Información, $\quad$ (33), 1-9. Recuperado de http://www.sociedadelainformacion.com/33/videos.pdf

Ruiz, F. y Escurra, L. (2013). Hábitos de consumo de Facebook y YouTube. Conciencia y estrategias metacognitivas en la lectura y estrategias de aprendizaje y estudio en universitarios. Persona, (16), 29-71. Recuperado de https://www.redalyc.org/articulo.oa?id=147131896002

Sato, A. (2012). The YouTube phenomenon: YouTube stars eliminating stereotypes in new media (tesis de maestría). Universidad del Sur de California.

Statista (2015). YouTube: Frecuencia de uso entre los usuarios España en 2015. Statista. Recuperado de https://es.statista.com/estadisticas/511381/frecuencia-de-uso-deyoutube-entre-los-usuarios-espana/

Tafadzwa Maziriri, E., Gapa, P. y Chuchu, T. (2020). Student Perceptions Towards the use of YouTube as An Educational Tool for Learning and Tutorials. International Journal of Instruction, 13(2), 119-138. Doi: 10.29333/iji.2020.1329a 

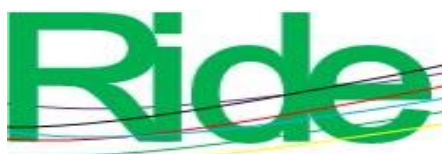

Revista Iberoamericana para la Investigación y el Desarrollo Educativo ISSN 2007 - 7467

Universidad de Guadalajara (UDG) (2017). Estadística Institucional 2016-2017. Recuperado de http://www.rectoria.udg.mx/sites/default/files/IA2016estadisticainstitucionalTBP.pdf

Yim, S., Sturzinger, E. and Lowrance, C. (16 de junio de 2019). Effects of YouTube Video as Pre-Lecture Preparation. 2019 ASEE Annual Conference \& Exposition. Retrieved from https://digitalcommons.usmalibrary.org/usma_research_papers/203/

Youtube (2019). Prensa. Recuperado de https://www.youtube.com/intl/es-419/about/press/ 